\title{
Pulmonary Embolism from Cement Augmentation of the Vertebral Body
}

\author{
Jose Manuel Fernando Ignacio $^{1,2}$, Katrina Hannah Dizon Ignacio ${ }^{3}$ \\ ${ }^{1}$ Department of Orthopedics, University of the Philippines Manila, Manila, Philippines \\ ${ }^{2}$ Institute of Orthopedics and Sports Medicine, St. Lukes Medical Center Global City, Taguig, Philippines \\ ${ }^{3}$ Department of Neurosciences, Philippine General Hospital, Manila, Philippines
}

Pulmonary cement embolism (PCE) can follow cement augmentation procedures for spine fractures due to osteoporosis, traumatic injuries, and painful metastatic lesions. PCE is underreported and it is likely that many cases remain undiagnosed. Risk factors for PCE have been identified, which can help alert clinicians to patients likely to develop the condition, and there are recommended techniques to reduce its incidence. Most patients with PCE are asymptomatic or only develop transient symptoms, although a few may exhibit florid cardiorespiratory manifestations which can ultimately be fatal. Diagnosis is mainly by radiographic means, commonly using simple radiographs and computed tomography scans of the chest with ancillary tests that assess the patient's cardiorespiratory condition. Management depends on the location and size of the emboli as well as the patient's symptomatology. The aim of this review is to raise awareness of the not uncommon complications of PCE following vertebral cement augmentation and the possibility of serious sequelae. Recommendations for the diagnosis and management of PCE are presented, based on the most recent literature.

Keywords: Pulmonary embolism; Bone cement; Polymethylmethacrylate; Kyphoplasty; Vertebroplasty

\section{Introduction}

Vertebral body cement augmentation, in the form of percutaneous kyphoplasty (PKP) and percutaneous vertebroplasty (PVP), is increasingly used in the management of painful vertebral fractures from osteoporosis and trauma, as well as for intractable pain arising from metastatic diseases of the vertebral column. An important benefit of these procedures is achieving the marked relief of symptoms through a truly minimally invasive technique. However, reports of complications are accumulating, including the embolization of cement into the pulmonary vascula- ture [1].

Although most cases of pulmonary cement embolism (PCE) remain asymptomatic and only require basic medical management, there have been reports of serious and fatal outcomes $[2,3]$. Recent studies have found that PCE is underreported [4]. It is therefore important that clinicians are aware of this potential complication following PVP and PKP. As yet, there are no guidelines on diagnosing PCE, and postprocedural chest imaging studies are not currently performed as standard. There are also no established guidelines as to the criteria for which patients should undergo further workup for possible PCE, what

Received Feb 22, 2017; Revised Jun 20, 2017; Accepted Jul 12, 2017

Corresponding author: Jose Manuel Fernando Ignacio

Spine Section, Department of Orthopedics, University of the Philippines Manila, Taft Avenue, Ermita, Manila, 1000 Metro Manila, Philippines

Tel: +63-25548466, Fax: +63-25276067, E-mail: kaingca@yahoo.com, kaingca@gmail.com 
that workup should comprise in addition to radiographic studies, or how PCE should be managed.

The aim of this review is to raise awareness of the potential for PCE following vertebral cement augmentation, and of the possible serious sequelae. Recommendations are suggested for establishing a diagnosis of PCE on the basis of its incidence, clinical presentation, risk factors, and prognosis, as well as for its management, based on the most recent literature.

\section{Materials and Methods}

The Cochrane, PubMed, and Medline online databases were searched for all articles available up to February 12, 2017. The online search of the Cochrane Library retrieved the title, abstracts, and keywords of papers using the search terms "pulmonary cement embolism" and "kyphoplasty" or "vertebroplasty"; this yielded three articles, all of which were trials. The online searches of the PubMed and Medline databases used all possible variations of terms for pulmonary cement emboli, including all combinations of the words "embolism," "emboli," and "embolus" and the words "polymethylmethacrylate," "PMMA," and "cement" with the terms "kyphoplasty" or "vertebroplasty"; this yielded an initial 133 articles. Articles in languages other than English were excluded. The titles, abstracts, and keywords of the articles were manually inspected for the following inclusion criteria: (1) studies pertaining to PCE after PKP or PVP, and (2) experimental or observational studies and systematic reviews. The included articles were extracted for review. Additional articles were then found from the bibliographies of certain studies. Data collection focused on the incidence, clinical characteristics, risk factors, diagnosis, and management of PCE following PKP or PVP.

\section{Results}

In total, 29 full articles were extracted and analyzed for the present review. In addition, a previous case report by one of the present authors was also included. Most of the included studies were observational or descriptive clinical studies. There were six case reports, one case control study, five prospective clinical trials, seven retrospective clinical trials, four systematic reviews, and one meta-analysis, as well as one in vitro study and one in vivo study.

\section{Discussion}

\section{Incidence of pulmonary cement embolism}

Only a few studies have documented the incidence of PCE arising from PVP and PKP. Case reports have claimed that the condition is rare $[5,6]$. The studies that investigated the incidence reported figures as low as $3.5 \%$ up to as high as $28.6 \%[7,8]$. This difference can be attributed to how actively the report authors searched for such complications and whether postoperative imaging was performed in all patients. When only postprocedural radiographs of the chest were obtained, the incidence varied from $1 \%$ to $6.8 \%$ [9]. However, if a more sensitive test such as chest computed tomography (CT) was employed, the incidence rose dramatically to the range $2.1 \%$ to $26 \%[2,4,10-12]$. Notably, the study documenting a low incidence of only $2.1 \%$, despite using chest CT scan as the diagnostic tool, obtained scans only when there was cement leakage visible on fluoroscopy [12]. It is likely that this protocol resulted in an underestimate of the true incidence of cement embolization $[2,4,10,11]$. In a retrospective study that assessed cement leakage noted on fluoroscopy during PVP, 23 of 244 patients (9.4\%) were found to have PCE on CT scans, but cement leakage was recognized by operators in only two of these 23 cases during the actual procedure. This suggests that, even for experienced practitioners, extravertebral cement leakage and PCE can be missed [13].

\section{Pulmonary cement embolism after vertebroplasty and kyphoplasty}

Several studies reported that PCE and local cement leakage were more common after PVP than after PKP $[14,15]$. One retrospective study found a significantly higher local leakage rate for PVP than for PKP [16]; however, further studies are needed to verify this statistical significance [15]. A meta-analysis that compared PVP and PKP in the treatment of vertebral compression fractures reported a higher prevalence of pulmonary embolus with PVP (33 of 3,601 cases [0.9\%] for PVP versus two of 565 cases [0.4\%] for PKP); however, this difference was not statistically significant, and the study did not distinguish between different kinds of emboli [17].

The reports of the in vitro and in vivo studies postulated reasons why cement extrusion is more frequently encountered after PVP. Intravertebral pressures measured during 


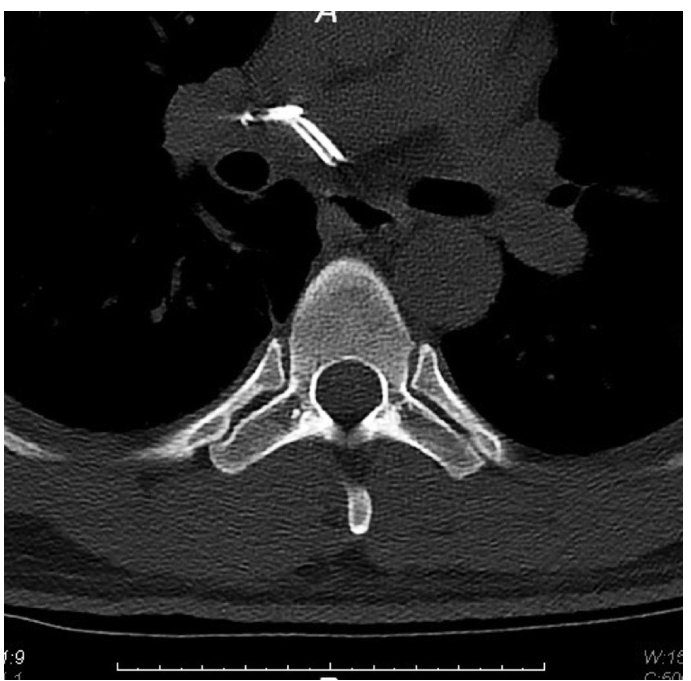

Fig. 1. Central type of pulmonary cement embolism.

cement augmentation showed that lower pressures were generated in PKP procedures than during PVP $[9,18]$. In addition, it has been suggested that the insertion of the balloon before cement infusion in PKP creates a cavity with a wall within the vertebral body. This may help contain the cement within the confines of the vertebra [17].

\section{Cement leakage and the risk factors for pulmonary cement embolism}

Recent reports have indicated that the pathway by which the cement leakage reaches the lungs starts from within the vertebral body, proceeding into the perivertebral venous system until reaching the azygos vein and the inferior vena cava, then continuing toward the right cardiac chambers or lodging in the pulmonary vasculature (Fig. 1) $[4,7,19]$. In addition, there have been reports of cement leakage into the spinal canal $[1,14]$. In a case report of a cement-augmented pedicle screw fixation procedure, finecut CT scans captured cement emanating from the nutrient vessels into the epidural space (Fig. 2) and from the perivertebral venous plexus before migrating centrally via the inferior vena cava toward the pulmonary vasculature (Fig. 3) [19].

Two prospective studies assessed the occurrence of PCE and found no statistically significant correlation in the development of PCE following PVP with factors such as the patient's age and sex, the number and level of the treated vertebrae, the volume of cement injected to each vertebra, the presence of intravertebral vacuum clefts, the use of uni- or bipedicular approaches, or the operators perform-

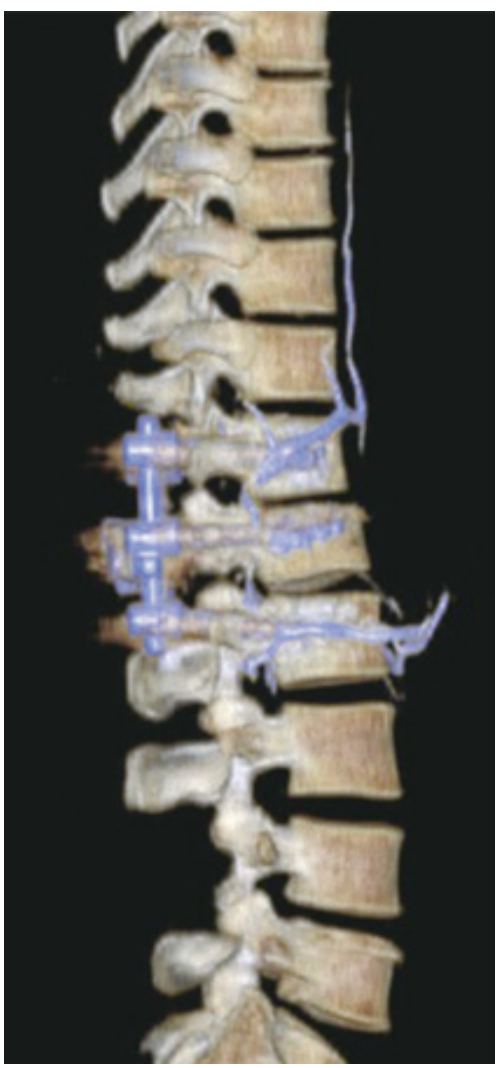

Fig. 2. Cement extravasation into nutrient vessels, the epidural space, and the paravertebral plexus of veins.

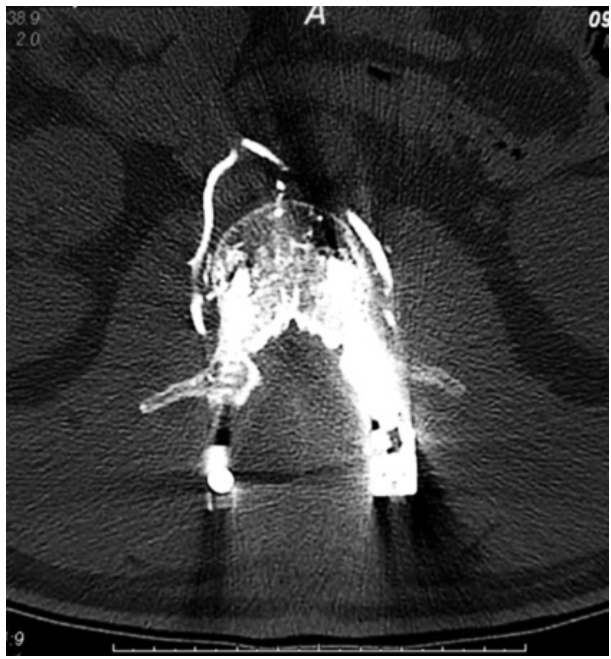

Fig. 3. Three-dimensional rendering of cement migration within the inferior vena cava and paraspinal venous plexuses.

ing the procedure (radiologist versus nonradiologist) $[4,12]$. It has been hypothesized by many researchers that using a larger volume of cement is a risk factor for cement leakage; however, other studies have confirmed that the occurrence of PCE is not associated with higher volumes 
of injected cement $[9,10,12]$. Risk factors identified as predictors of cement embolism into the pulmonary vasculature include the presence of cement in the azygos vein or in the inferior vena cava $[4,12]$. A similar, retrospective study on the occurrence of PCE following PVP and PKP found an association between PCE and leakage of the cement into the paravertebral venous plexus. Again, there was no statistically significant correlation between PCE and the number of vertebral bodies treated surgically [11]. Another study, however, found that PCE occurred more frequently in the patients with a greater total number of treated vertebral levels [20].

A study on patients who underwent PVP reported that, in those treated for compression fractures, the incidence of PCE did not vary with the etiology of fractures, whether due to osteoporosis, trauma, or malignancies [12]. This contradicted other studies that claimed that PCE occurred more frequently with the treatment of some malignant lesions that cause greater cortical destruction of the vertebral body and have a higher vascularity $[4,9,11]$. Fracture pattern and the area of vertebral destruction have also been related to an increased risk for cement leakage. In addition, end plate disruption, anterior or posterior wall fractures, and posterior cortical wall destruction can increase the risk of cement leakage [17]. More studies are needed to verify these risk factors and predictors for PCE following cement augmentation.

\section{Techniques to minimize pulmonary cement embo- lism}

Previous reports have suggested that using lower-viscosity PMMA increases the risk of cement leakage into perivertebral veins [9,7], as does higher pressure applied during the injection of the cement $[1,21]$. Early injection of cement in the liquid phase and injection too quickly into the vertebral body also pose risks of the relevant extravasation $[2,9]$. Recommendations to help reduce this risk include limiting the volume of cement injected, ensuring a viscous consistency or sufficient polymerization of PMMA prior to injection, and the immediate termination of cement injection if venous leakage is observed. However, PMMA viscosity is usually judged subjectively by waiting for the cement to reach a consistency similar to that of toothpaste. This is less accurate than using viscometers and other specialized equipment; conversely, using this equipment may be too time-consuming. Some studies have recommended injecting cement in stages, stopping after injecting a small volume of cement, even if leakage is not observed, and continuing after a 20-30 seconds interval $[1,14]$. Some have suggested intravertebral venography prior to the PMMA injection or injection of sclerosing agents into the vertebral bodies to close the venous channels; however, their use is controversial $[14,21,22]$.

It has been proposed that cement leakage is operatordependent, with less leakage occurring with physicians or radiologists who are more experienced and careful in filling vertebral bodies. The need for meticulous monitoring with guidance equipment has also been stressed. Although uniplanar fluoroscopy is more common, it can be difficult to view real-time lateral vertebral leakage of cement due to the overlap of the cement located within and the cement leaking lateral to the vertebral body. This is best documented in the anteroposterior (AP) view. However, when using uniplanar fluoroscopy, the preferred view during PMMA injection is the lateral projection. Biplanar or intermittent AP fluoroscopy, and even threedimensional fluoroscopy, could be used to overcome this problem $[1,4]$. These propositions, however, have yet to be substantiated by formal studies.

A few studies have reported that lavage of a vertebral body prior to cement injection to provide a better filling pattern, and the use of a vent hole technique to reduce vertebral pressure during cement injection, reduced the likelihood of overall cement leakage compared to control $[23,24]$. Interestingly, a study in which a group of patients were ventilated with a higher positive end-expiratory pressure of $15 \mathrm{~cm} \mathrm{H}_{2} \mathrm{O}$ during cement injection showed significantly less local cement leakage, as well as a lower incidence of PCE compared to patients ventilated without the increased positive expiratory end pressure [7]. However, the findings of these studies have yet to be confirmed.

\section{Cement types used in the procedures}

Cement augmentation has become widely used in vertebral procedures over the past two decades. During the earlier periods of vertebral cement augmentation, cement products designed for hip and knee arthroplasty were adopted for use in PVP. These cement products required high polymerization temperatures of up to $100^{\circ} \mathrm{C}$. The high temperatures posed an increased risk for thermal injury and necrosis, making cement leakage more likely. Later, PMMA with lower polymerization temperatures 
was developed, greatly reducing these complications. PMMA has the advantage of having good handling, injectability, and immediate load capacity. Newer varieties of bone cement are in developments that have greater viscosity and lower stiffness properties, with the aim of reducing these complications even further [7].

Cement mixtures were not standardized until the late 2000s. In a retrospective study, Luetmer et al. [13] noted that cement mixtures without standardized PMMA-tosolvent ratios were used in their institution before 2005, with bone cement with fixed proportions of PMMA to barium sulfate used subsequently. A similar issue was reported by Choe et al. [11], who noted in a study that ran from 2000 to 2002 that the addition of barium to their cement mixture was not standardized. Thus, the use of different cement products, inconsistencies in the composition of cement mixtures, and differences in the amount of cement injected intraoperatively could also account for the variability in the incidence of PCE across studies. The incidence of PCE may also vary between studies because of different patient selection criteria and variations in operative technique.

\section{Clinical presentation and outcomes}

Most patients with PCE remain asymptomatic [2,4,9,12,25]. This may explain why patients are not routinely screened with postoperative chest imaging [9]. Results from VERTOS II, a prospective multicenter randomized controlled trial that compared PVP with conservative therapy, showed that all the patients who developed PCE following PVP remained asymptomatic after one year. The emboli ranged from 1 to $12 \mathrm{~mm}$ in size and were randomly distributed in the lung periphery, with no specific lobar distribution [12]. Similar findings were noted for the two cases of PCE detected in a study that enrolled 616 patients who underwent PVP; the areas of emboli spanned a few millimeters and were described as minimal [26].

Other studies on PCE after PVP or PKP have also reported emboli that were small and peripheral, with almost all patients remaining asymptomatic $[4,13]$. It has been reported that pulmonary cement deposits remained morphologically unchanged, even one year after the procedure [26]. One systematic review of the management of PCE after PVP and PKP found 24 published studies that reported symptomatic PCEs, with the most common symptom being dyspnea. In most cases, symptoms were transient or lasted for only a short period of time [8]. One study reported that symptoms related to pulmonary embolism were observed in only a small percentage ( $0.4 \%$ to 0.9\%) of cases of PCE after PVP and PKP [14].

Aside from dyspnea, symptoms of PCE include precordial chest pain or tightness, coughing, hemoptysis, dizziness, sweating, and palpitation. Physical examination may reveal cyanosis, tachypnea, hypotension, irregular cardiac rhythm, or cardiac arrest [27]. Laboratory findings may show increased plasma D-dimer levels, low oxygen saturation, hypocapnia, and ventilation/perfusion mismatch $[1,9]$. Most symptoms begin days to weeks after the procedure. However, symptoms may be observed even during the PVP or PKP procedure itself. There have also been cases in which patients developed symptoms months, and even as long as 10 years, after the procedure $[6,20,28]$. Fatal cardiopulmonary complications of PCE have also been reported, including acute respiratory distress syndrome, pulmonary infarction, hypercapnia, cardiac arrest, and death $[3,4]$.

\section{Diagnosis of pulmonary cement embolism}

PCE is probably the complication of vertebral cement augmentation procedures that causes the greatest concern. In most instances, it is asymptomatic and may go undiagnosed [20]. As yet, there is no established protocol for its diagnosis. The most common imaging procedures used are chest radiography and chest CT. Some authors have recommended acquiring chest radiographs within the first 24 hours after PVP or PKP, claiming that the incidence and risk for PCE justifies standardized screening. This would be of benefit for the early detection of the presence of embolism [20]. The use of CT scan as a routine screening tool is in question. CT has a higher sensitivity for PCE detection but also exposes patients to more radiation [9].

Unlike classic pulmonary thromboemboli, polymethylmethacrylate cement has a high density compared to that of the lung parenchyma. This allows for the easy recognition and visualization of cement emboli on conventional chest radiographs [1]. Studies have reported PCE as solitary or multiple, tubular or branching, radiodense lines in the lungs on chest radiographs [4,11]. Also suggestive of PCE on chest radiography are areas of high-density opacity in a tubular branching pattern that correspond to the distribution of arteries [26,29]. In a study in which chest CT scans were used to document the presence of PCE, 
the detected PCEs were also evident on plain radiographs. The authors concluded that the thorough study of good quality radiographs can be as good as chest CT scans for the detection of PCE [11].

Notably, CT has been reported as an extremely effective modality for the diagnosis of PCE. Unenhanced CT is commonly used without the need for an intravenous contrast medium because of the hyperdense nature of the cement deposits. In symptomatic patients, however, some authors have suggested that contrast-enhanced CT may be of greater value because it may be able to demonstrate endovascular thrombi [11].

In a prospective study of PCE after PVP in vertebral compression fractures, specific criteria were used to differentiate a PCE from a calcified granuloma; in some circumstances, these can be difficult to discriminate on CT. The presence of high-attenuation signals smaller than the diameter of the lumen of the pulmonary artery was highly suggestive of an intraluminal embolus. Where there are past radiographic imaging results available for comparison, a newly developed branching high-attenuation area would also be part of the criteria for considering PCE $[4,25]$. This may be important because most patients who undergo cement augmentation procedures are likely to have had a preprocedure chest radiograph as part of routine medical clearance or evaluation in preparation for the procedure; this would allow the postprocedure chest radiograph to be compared with the preprocedure study. Other adjuvant tests include transesophageal echocardiography to evaluate for secondarily elevated pulmonary artery pressure and hemodynamic instability, and pulmonary function tests to evaluate variations in lung diffusion capacity.

\section{Management of pulmonary cement embolism}

Because only a limited number of reports related to PCE have been published, there is no clear therapeutic protocol that has been accepted as a standard for treatment. Recent reports, however, suggest that the recommended management approach should be based on the severity of the patient's symptoms and the location and size of the emboli. Accordingly, patients with PCE can be divided into four groups: (1) asymptomatic peripheral embolism, (2) symptomatic peripheral embolism, (3) asymptomatic central embolism, and (4) symptomatic central embolism. Central embolism is defined as involving the main pul- monary trunk and/or the right or left main pulmonary arteries; any embolism beyond that median is considered a peripheral pulmonary embolism. A similar approach to management has been taken in several studies. For patients in group 1, with asymptomatic peripheral PCEs, it is recommended there should be no treatment. It remains unclear whether systemic anticoagulation is beneficial for this patient group and whether treatment is justified. Thus, conservative treatment, observation, and regular clinical follow-up are recommended. For those in groups 2 and 3, with symptomatic peripheral or asymptomatic central embolisms, the recommendation is to follow the standard treatment guidelines for the treatment of thrombotic pulmonary embolisms, including initial heparinization followed by full anticoagulation with warfarin therapy for 3-6 months. Finally, for group 4 patients with symptomatic central embolisms, surgical treatment with embolectomy has been suggested $[8,9,21,30]$.

\section{Conclusions}

The incidence of PCE following vertebral cement augmentation procedures is higher than previously thought. Although it has been reported as being a more frequent complication of PVP than of PKP, further studies are needed to determine the statistical significance of this difference. Techniques to minimize the likelihood of cement leakage and subsequent embolization have been described. Although PCE is mostly asymptomatic, clinicians should be aware of the possibility of respiratory manifestations that can occur as early as periprocedurally to as late as years after the vertebral augmentation procedure. Apart from vena cava and azygos leakage, predictors and risk factors for PCE have yet to be identified. Routine postoperative chest radiography appears to be justifiable and is recommended given the higher than expected incidence of PCE and, although rare, its potentially fatal outcome. The likelihood that preprocedural chest radiographs are available for comparison increases the usefulness of this recommendation. Reasonable suspicion, such as when a patient manifests with respiratory symptoms and unstable vital signs, as well as periprocedural cement leakage, warrant a CT scan. There is agreement that approaches to the management of PCE should be based on the patient's symptoms and the location of the embolus, but standardized protocols for the diagnostic and therapeutic management of PCE have yet to be established. It 
is hoped that these become available in the near future, given the growing body of knowledge on PCE, a complication of vertebral cement augmentation that was once thought uncommon.

\section{Conflict of Interest}

No potential conflict of interest relevant to this article was reported.

\section{References}

1. Wang LJ, Yang HL, Shi YX, Jiang WM, Chen L. Pulmonary cement embolism associated with percutaneous vertebroplasty or kyphoplasty: a systematic review. Orthop Surg 2012;4:182-9.

2. Venmans A, Lohle PN, van Rooij WJ, Verhaar HJ, Mali WP. Frequency and outcome of pulmonary polymethylmethacrylate embolism during percutaneous vertebroplasty. Am J Neuroradiol 2008;29:1983-5.

3. Sinha N, Padegal V, Satyanarayana S, Santosh HK. Pulmonary cement embolization after vertebroplasty, an uncommon presentation of pulmonary embolism: a case report and literature review. Lung India 2015;32:602-5.

4. Kim YJ, Lee JW, Park KW, et al. Pulmonary cement embolism after percutaneous vertebroplasty in osteoporotic vertebral compression fractures: incidence, characteristics, and risk factors. Radiology 2009;251:250-9.

5. Lamparello NA, Jaswani V, DeSousa K, Shapiro M, Kovacs S. Percutaneous retrieval of an embolized kyphoplasty cement fragment from the pulmonary artery: a case report and literature review. J Radiol Case Rep 2016;10:40-7.

6. Rothermich MA, Buchowski JM, Bumpass DB, Patterson GA. Pulmonary cement embolization after vertebroplasty requiring pulmonary wedge resection. Clin Orthop Relat Res 2014;472:1652-7.

7. El Saman A, Kelm A, Meier S, et al. Intraoperative PEEP-ventilation during PMMA-injection for augmented pedicle screws: improvement of leakage rate in spinal surgery. Eur J Trauma Emerg Surg 2013;39:461-8.

8. Krueger A, Bliemel C, Zettl R, Ruchholtz S. Management of pulmonary cement embolism after percuta- neous vertebroplasty and kyphoplasty: a systematic review of the literature. Eur Spine J 2009;18:1257-65.

9. Bliemel C, Buecking B, Struewer J, Piechowiak EI, Ruchholtz S, Krueger A. Detection of pulmonary cement embolism after balloon kyphoplasty: should conventional radiographs become routine? Acta Orthop Belg 2013;79:444-50.

10. Anselmetti GC, Corgnier A, Debernardi F, Regge D. Treatment of painful compression vertebral fractures with vertebroplasty: results and complications. Radiol Med 2005;110:262-72.

11. Choe DH, Marom EM, Ahrar K, Truong MT, Madewell JE. Pulmonary embolism of polymethyl methacrylate during percutaneous vertebroplasty and kyphoplasty. Am J Roentgenol 2004;183:1097102.

12. Venmans A, Klazen CA, Lohle PN, et al. Percutaneous vertebroplasty and pulmonary cement embolism: results from VERTOS II. Am J Neuroradiol 2010;31:1451-3.

13. Luetmer MT, Bartholmai BJ, Rad AE, Kallmes DF. Asymptomatic and unrecognized cement pulmonary embolism commonly occurs with vertebroplasty. Am J Neuroradiol 2011;32:654-7.

14. Habib N, Maniatis T, Ahmed S, et al. Cement pulmonary embolism after percutaneous vertebroplasty and kyphoplasty: an overview. Heart Lung 2012;41:50911.

15. Zhang JD, Poffyn B, Sys G, Uyttendaele D. Comparison of vertebroplasty and kyphoplasty for complications. Orthop Surg 2011;3:158-60.

16. Lee IJ, Choi AL, Yie MY, et al. CT evaluation of local leakage of bone cement after percutaneous kyphoplasty and vertebroplasty. Acta Radiol 2010;51:64954.

17. Eck JC, Nachtigall D, Humphreys SC, Hodges SD. Comparison of vertebroplasty and balloon kyphoplasty for treatment of vertebral compression fractures: a meta-analysis of the literature. Spine J 2008;8:488-97.

18. Weisskopf M, Ohnsorge JA, Niethard FU. Intravertebral pressure during vertebroplasty and balloon kyphoplasty: an in vitro study. Spine (Phila Pa 1976) 2008;33:178-82.

19. Ignacio JM, Ignacio SD. Pulmonary bone cement embolism following pedicle screw instrumentation with bone cement augmentation for a burst fracture of 
L1 in a non-osteoporotic spine. Acta Medica Philipp 2013;47:76-9.

20. Bopparaju S, Varon J, Surani S. Pulmonary embolism with vertebral augmentation procedures. Case Rep Pulmonol 2013;2013:785307.

21. Duran C, Sirvanci M, Aydogan M, Ozturk E, Ozturk C, Akman C. Pulmonary cement embolism: a complication of percutaneous vertebroplasty. Acta Radiol 2007;48:854-9.

22. Potet J, Weber-Donat G, Curis E, et al. Incidence of pulmonary cement embolism after real-time CT fluoroscopy-guided vertebroplasty. J Vasc Interv Radiol 2013;24:1853-60.

23. Benneker LM, Heini PF, Suhm N, Gisep A. The effect of pulsed jet lavage in vertebroplasty on injection forces of polymethylmethacrylate bone cement, material distribution, and potential fat embolism: a cadaver study. Spine (Phila Pa 1976) 2008;33:E90610.

24. Hoppe S, Elfiky T, Keel MJ, Aghayev E, Ecker TM, Benneker LM. Lavage prior to vertebral augmentation reduces the risk for cement leakage. Eur Spine J 2016;25:3463-9.
25. Saracen A, Kotwica Z. Complications of percutaneous vertebroplasty: an analysis of 1100 procedures performed in 616 patients. Medicine (Baltimore) 2016;95:e3850.

26. Trumm CG, Pahl A, Helmberger TK, et al. CT fluoroscopy-guided percutaneous vertebroplasty in spinal malignancy: technical results, PMMA leakages, and complications in 202 patients. Skeletal Radiol 2012;41:1391-400.

27. Health Quality Ontario. Percutaneous vertebroplasty for treatment of painful osteoporotic vertebral compression fractures: an evidence-based analysis. Ont Health Technol Assess Ser 2010;10:1-45.

28. Leitman $\mathrm{D}, \mathrm{Yu} \mathrm{V}$, Cox C. Investigation of polymethylmethacrylate pulmonary embolus in a patient ten years following vertebroplasty. J Radiol Case Rep 2011;5:14-21.

29. Nooh A, Abduljabbar FH, Abduljabbar AH, Jarzem P. Pulmonary artery cement embolism after a vertebroplasty. Case Rep Orthop 2015;2015:582769.

30. Toru U, Coskun T, Acat M, Onaran H, Gul S, Cetinkaya E. Pulmonary cement embolism following percutaneous vertebroplasty. Case Rep Pulmonol 2014;2014:851573. 